\title{
GENERALIZED CHARACTER SUMS ASSOCIATED TO REGULAR PREHOMOGENEOUS VECTOR SPACES
}

\author{
DAVID KAZHDAN AND ALEXANDER POLISHCHUK
}

The purpose of this note is to give a short derivation of the finite field analogue of Sato's functional equation for the zeta function associated with a prehomogeneous vector space (see 16). We restrict ourselves to the case of a regular prehomogeneous vector space, however, we allow to twist our character sums by local systems associated to arbitrary representations of the component group of the stabilizer of a generic point. The main idea of our approach is to use the Picard-Lefschetz formula in $l$-adic cohomology instead of using a lift of a prehomogeneous space to the characteristic zero (as it is done in [4]). Also we deduce another functional equation associated with a regular prehomogeneous vector space (theorem 1.4).

\section{Preliminaries and Formulation of the main ReSUlts}

Throughout this paper $k$ denotes an algebraically closed field of characteristic $p$, $k_{0}$ denotes its finite subfield. By a sheaf on a scheme $X$ over $k$ or $k_{0}$ we mean an object of the derived category of constructible $l$-adic sheaves, where $l \neq p$ (see e.g. 2]). For a morphism $f: X \rightarrow Y$ we denote by $f_{!}, f_{*}, f^{!}, f^{*}$ the natural derived functors between the sheaves on $X$ and $Y$. Often we use the notation $F(f)$ for $f^{*} F$ where $F$ is a sheaf on $Y$. For a sheaf $F$ we denote by ${ }^{p} H^{i} F$ the $i$-th perverse cohomology of $F$ (see [1]). For a scheme $X$ defined over $k_{0}$ we denote by Frob $k_{k_{0}}$ the geometric Frobenius morphism on $X$. Recall that according to theorem of Laumon (see [11) if $F$ is a sheaf on a $k$-scheme $X$ then one has the equality of two kinds of Euler-Poincaré characteristics

$$
\chi(X, F)=\chi_{c}(X, F)
$$

By a multiplicative character of $k$ we always mean a continuous homomorphism from $\hat{\mathbb{Z}}(1)(k)=$ proj $\lim _{(N, p)=1} \mu_{N}(k)$ to $\overline{\mathbb{Q}}_{l}^{*}$. For every multiplicative character $\chi$ we denote by $L_{\chi}$ the corresponding Kummer sheaf on $\mathbb{G}_{m}$. We will often use the following theorem of Deligne (see [6]): for every sheaf $K$ on $\mathbb{G}_{m}$ and every multiplicative character $\chi$ one has $\chi\left(\mathbb{G}_{m}, L_{\chi} \otimes K\right)=\chi\left(\mathbb{G}_{m}, K\right)$.

If $\chi$ is a character of $k_{0}^{*}$ then it can be considered as a multiplicative character of $k$ via the natural homomorphism $\hat{\mathbb{Z}}(1)(k) \rightarrow k_{0}^{*}$. Then the sheaf $L_{\chi}$ is defined over $k_{0}$ and Frob $k_{0}$ acts on the fiber of $L_{\chi}$ at $a \in \mathbb{G}_{m}\left(k_{0}\right)$ as the scalar $\chi(a)$.

If $G$ is a connected algebraic group acting on $X$ then one can define the category of $G$-equivariant perverse sheaves on $X$ (see e.g. [13). More generally, one can consider relative $G$-equivariant perverse sheaves on $X$ corresponding to the pair $(\alpha, \chi)$ where $\alpha: G \rightarrow \mathbb{G}_{m}$ is a group homomorphism, $\chi$ is a multiplicative character of $k$. These are perverse sheaves $F$ such that there exists an isomorphism

$$
m^{*} F[\operatorname{dim} G] \simeq L_{\chi}(\alpha) \otimes F[\operatorname{dim} G]
$$

Both authors are partially supported by NSF grants. 
of perverse sheaves on $G \times X$, where $m: G \times X \rightarrow X$ is the action morphism (such an isomorphism on $G \times X$ can be chosen canonically by imposing the condition that its restriction to $e \times X$ is the identity, where $e \in G$ is the neutral element). If the action of $G$ on $X$ is transitive, and $F$ is a relative $G$-equivariant perverse sheaf on $X$ then there exists a local system $\mathcal{L}$ on $X$ such that $F=\mathcal{L}[\operatorname{dim} X]$. One can show that a subquotient of a relative $G$-equivariant perverse sheaf is again relative $G$-equivariant (the proof is similar to [14],(1.9.1)). In particular, an irreducible object in the category of relative $G$-equivariant perverse sheaves is irreducible as a perverse sheaf.

Let $\psi$ be a non-trivial additive character of $k_{0}$. We denote by $L_{\psi}$ the corresponding Artin-Schreier sheaf on $\mathbb{A}^{1}$ (defined over $k_{0}$ ). Let $V$ be a finite-dimensional vector space over $k$. Then the Fourier-Deligne transform (see [12]) of a sheaf $F$ on $V$ is the sheaf

$$
\mathcal{F}(F)=\left(p_{2}\right) !\left(p_{1}^{*} F \otimes L_{\psi}\left(\left\langle v, v^{\vee}\right\rangle\right)\right)[\operatorname{dim} V]
$$

on the dual space $V^{\vee}$, where $p_{i}$ are the projections of $V \times V^{\vee}$ on two factors, $\left\langle v, v^{\vee}\right\rangle$ denotes the pairing between $V$ and $V^{\vee}$.

For a multiplicative character $\chi$ we denote

$$
G(\chi)=G(\chi, \psi)=H_{c}^{1}\left(\mathbb{G}_{m}, L_{\chi} \otimes L_{\psi}\right) .
$$

This is a 1-dimensional $\overline{\mathbb{Q}}_{l}$-space. If $\chi$ is a character of $k_{0}^{*}$ then the action of the Frobenius on $G(\chi)$ is given by

$$
g(\chi)=g(\chi, \psi)=-\sum_{a \in k_{0}^{*}} \chi(a) \psi(a) .
$$

One has the following isomorphism of sheaves on $\mathbb{G}_{m}$ :

$$
j_{1}^{*} \mathcal{F}\left(j_{1 !} L_{\chi}\right) \simeq G(\chi) \otimes L_{\chi^{-1}}
$$

where $j_{1}: \mathbb{G}_{m} \rightarrow \mathbb{A}^{1}$ is the standard embedding. Furthermore, if $\chi$ is non-trivial then

$$
\mathcal{F}\left(j_{1 !} L_{\chi}\right) \simeq G(\chi) \otimes j_{1 !} L_{\chi^{-1}} .
$$

The facts about prehomogeneous spaces we use below are essentially contained in [10, we only need to make slight changes due to finite characteristics (see e.g. section 1 of [9]). Let $G$ be a connected reductive group over $k, V$ be a regular prehomogeneous vector space over $k$ for $G$. This means that $G$ acts linearly on $V$ with an open orbit $U \subset V$ and there is a relative invariant $f \in k[V]$ with the character $\alpha \in \operatorname{Hom}\left(G, \mathbb{G}_{m}\right): f(g x)=\alpha(g) f(x)$, such that $F=\operatorname{grad} \log f$ gives a dominant map from $U$ to $V^{\vee}$. We fix such an invariant $f$ once and for all. Often we consider $f$ as a morphism $U \rightarrow \mathbb{G}_{m}$, in particular, we consider sheaves of the form $L_{\chi}(f)$ on $U$ where $\chi$ is a multiplicative character. The dual space $V^{\vee}$ is also a regular prehomogeneous space for $G, F$ induces a $G$-equivariant morphism of $U$ onto an open $G$-orbit $U^{\vee} \subset V^{\vee}$. We assume that our prehomogeneous vector space is separable in the sense of [9], i.e. the map $F$ is separable and the open orbit morphism $G \rightarrow V^{*}: g \mapsto g v^{\vee}$ is separable for generic $v^{\vee}$. Then as shown in [9] there exists a relative invariant $f^{\vee} \in k\left[V^{\vee}\right]$ with the character $\alpha^{-1}$ and the map $F^{\vee}=\operatorname{grad} \log f^{\vee}$ is inverse to $F$. Moreover, in this case the closed set $V \backslash U$ (resp. $V^{\vee} \backslash U^{\vee}$ ) coincides with the hypersurface $f=0$ (resp. $f^{\vee}=0$ ). Since the function 
$f^{\vee}(F(x)) f(x)$ on $U$ is $G$-invariant it should be constant, hence, we can rescale $f^{\vee}$ in such a way that

$$
f^{\vee}(F(x))=f(x)^{-1} .
$$

Substituting $x=F^{\vee}\left(x^{\vee}\right)$ into this equation we obtain

$$
f\left(F^{\vee}\left(x^{\vee}\right)\right)=f^{\vee}(x)^{-1} .
$$

Since $F$ is homogeneous of degree -1 the equation (1.3) also implies that $\operatorname{deg} f^{\vee}=$ $\operatorname{deg} f$.

Let $H$ be the stabilizer of a point $x_{0} \in U$. Then $G$-equivariant local systems on $U$ correspond to representations of the group $\bar{H}$ of connected components of $H$. For an irreducible representation $\rho$ of $\bar{H}$ let us denote by $\mathcal{V}_{\rho}$ the corresponding local system on $U$.

For a multiplicative character $\chi$ and an irreducible $\bar{H}$-representation $\rho$ we consider the relative $G$-equivariant perverse sheaf

$$
S_{V}(\chi, \rho)=j_{!}\left(L_{\chi}(f) \otimes \mathcal{V}_{\rho}\right)[n]
$$

on $V$ where $j: U \rightarrow V$ is the natural open embedding, $n=\operatorname{dim} V$.

We want to determine the Fourier-Deligne transform of the perverse sheaf $S_{V}(\chi, \rho)$ when it is irreducible. We'll show below that this is the case for almost all $\chi$. Also we'll show that the restriction of $\mathcal{F}\left(S_{V}(\chi, \rho)\right)$ to $U^{\vee}$ is non-zero, therefore, by irreducibility $\mathcal{F}\left(S_{V}(\chi, \rho)\right)$ is the Goresky-MacPherson extension of its restriction to $U^{\vee}$ which should be of the form $L_{\chi^{-1}}\left(f^{\vee}\right) \otimes \mathcal{V}^{\vee}[n]$ for some irreducible $G$-equivariant sheaf $\mathcal{V}^{\vee}=\mathcal{V}_{\rho^{\vee}}$ on $U^{\vee}$. It follows that for generic $\chi$ one has

$$
\mathcal{F}\left(S_{V}(\chi, \rho)\right) \simeq S_{V^{\vee}}\left(\chi^{-1}, \rho^{\vee}\right) .
$$

Our main problem will be to determine $\rho^{\vee}$. We'll show that it doesn't depend on $\chi$ but only on $\rho$. Furthermore, notice that there is a natural isomorphism between the stabilizer $H$ of a point $x \in U$ and the stabilizer of the point $F(x) \in U^{\vee}$ (since $F$ is $G$-equivariant). Thus, $\mathcal{V}^{\vee}$ corresponds to some irreducible representation of $\bar{H}$ depending only on the original representation $\rho$. The explicit form of this dependence it given in the following theorem.

Theorem 1.1. There exists a homomorphism $\epsilon: \bar{H} \rightarrow\{ \pm 1\}$ such that

(a) for almost all multiplicative characters $\chi$ one has

$$
\mathcal{F}\left(S_{V}(\chi, \rho)\right) \simeq S_{V^{\vee}}\left(\chi^{-1}, \epsilon \otimes \rho\right) .
$$

(b) for all characters $\chi$ one has

$$
\left(j^{\vee}\right)^{*} \mathcal{F}\left(S_{V}(\chi, \rho)\right) \simeq\left(j^{\vee}\right)^{*} S_{V^{\vee}}\left(\chi^{-1}, \epsilon \otimes \rho\right) .
$$

Now assume that our prehomogeneous vector space and a relative invariant $f$ are defined over a finite field $k_{0}$ and assume that $U\left(k_{0}\right)$ is non-empty (then $f^{\vee}$ is also defined over $\left.k_{0}\right)$. Then for every character $\chi$ of $k_{0}^{*}$ the sheaves $S_{V}(\chi, \rho)$ can be equipped with the natural action of Frobenius but the isomorphism (1.6) is not in general compatible with the action of Frobenius on LHS and RHS. The difference between two actions is given by a non-zero constant depending on $\chi$. We will show that this constant is essentially a product of some Gauss sums. Then considering the traces of Frobenius acting on both sides of (1.6) one derives some identities for character sums. Here is a more precise formulation. Let us fix a point $x_{0} \in U\left(k_{0}\right)$. Then there is a natural action of Frob $_{k_{0}}$ on the component group $\bar{H}$. Let $\rho$ be an irreducible representation of $\bar{H}$ in a $\overline{\mathbb{Q}}_{l}$-space $W$. 
Definition. An action of $\operatorname{Frob}_{k_{0}}$ on the representation $\rho$ is an operator $A \in \mathrm{GL}(W)$ such that

and $A^{N}=$ id for some $N>0$

$$
\rho\left(\operatorname{Frob}_{k_{0}}(h)\right)=A^{-1} \rho(h) A
$$

Remark. An action of $\operatorname{Frob}_{k_{0}}$ on $\rho$ exists if and only if $\bar{H}$-representations $\rho \circ$ Frob $_{k_{0}}$ and $\rho$ are isomorphic.

Let us fix an action of $\operatorname{Frob}_{k_{0}}$ on $\rho$. Then $A$ descends to an isomorphism of $G$-equivariant local systems Frob $_{k_{0}}^{*} \mathcal{V}_{\rho} \rightarrow \mathcal{V}_{\rho}$. Notice that our assumption $A^{N}=$ id implies that the Weil sheaf $\mathcal{V}_{\rho}$ is pure of weight 0 . For every finite extension $k_{1}$ of $k_{0}$ and $x \in U\left(k_{1}\right)$ we define a conjugacy class $\rho_{x}$ in $\mathrm{GL}(W)$ as follows: choose an element $g \in G / H_{0}\left(\overline{k_{0}}\right)$ such that $g x_{0}=x$ (where $H_{0}$ is the identity component of $H)$, then $\operatorname{Frob}_{k_{1}}(g)=g h$ for some $h \in H / H_{0}=\bar{H}$, now $\rho_{x}$ is the conjugacy class of $A^{\left[k_{1}: k_{0}\right]} \rho\left(h^{-1}\right)$. Then we have

$$
\operatorname{Tr}\left(\operatorname{Frob}_{k_{1}},\left(\mathcal{V}_{\rho}\right)_{x}\right)=\operatorname{Tr}\left(\rho_{x}\right) .
$$

We apply similar constructions to the dual prehomogeneous space $V^{\vee}$ taking as a fixed point $x_{0}^{\vee}=F\left(x_{0}\right) \in U^{\vee}\left(k_{0}\right)$. Also we fix a square root of $\left|k_{0}\right|$ in $\overline{\mathbb{Q}}_{l}^{*}$. This leads to a choice of a square root of $\left|k_{1}\right|$ for every finite extension $k_{0} \subset k_{1}$. Recall that an algebraic number $a \in \overline{\mathbb{Q}}_{l}^{*}$ is called pure of weight $w$ relative to $\left|k_{0}\right|$ if for any isomorphism $i: \overline{\mathbb{Q}}_{l} \rightarrow \mathbb{C}$ one has $|i(a)|=\left|k_{0}\right|^{w / 2}$.

Theorem 1.2. For every irreducible representation $\rho$ of $\bar{H}$ equipped with an action of Frob $k_{0}$ there exists a finite extension $k_{0}^{\prime}$ of $k_{0}$, two collections of characters of $\left(k_{0}^{\prime}\right)^{*}:\left(\lambda_{1}, \ldots, \lambda_{m+d}\right)$ and $\left(\mu_{1}, \ldots, \mu_{m}\right)$, where $d=\operatorname{deg}(f)$, and a constant $\zeta \in \overline{\mathbb{Q}}_{l}^{*}$ of weight 0 , such that for every finite extension $k_{1}$ of $k_{0}^{\prime}$ and for every character $\chi$ of $k_{1}^{*}$ one has

$$
\begin{array}{r}
(-1)^{n} q^{-\frac{n}{2}} \sum_{x \in U\left(k_{1}\right)} \operatorname{Tr}\left(\rho_{x}\right) \chi(f(x)) \psi\left(T\left\langle x, x^{\vee}\right\rangle\right)=\zeta^{\left[k_{1}: k_{0}\right]} \times \\
\prod_{i=1}^{m+d} \frac{g\left(\chi\left(\lambda_{i} \circ N\right)\right)}{\sqrt{q}} \cdot \prod_{j=1}^{m} \frac{g\left(\chi^{-1}\left(\mu_{j} \circ N\right)\right)}{\sqrt{q}} \cdot \operatorname{Tr}\left((\rho \otimes \epsilon)_{x^{\vee}}\right) \chi^{-1}\left((-1)^{m} f^{\vee}\left(x^{\vee}\right)\right)
\end{array}
$$

for every $x^{\vee} \in U^{\vee}\left(k_{1}\right)$, where $q=\left|k_{1}\right|, T: k_{1} \rightarrow k_{0}$ is the trace, $N: k_{1}^{*} \rightarrow\left(k_{0}^{\prime}\right)^{*}$ is the norm. Furthermore, if $\chi$ is different from all the characters $\lambda_{i}^{-1} \circ N$ and $\mu_{j} \circ N$ then the sum in the LHS vanishes for $x^{\vee}$ in the complement to $U^{\vee}$.

Remarks. 1. If all the characters $\lambda_{i}$ and $\mu_{j}$ are compositions of the norm homomorphism and some characters of a subextension $k_{0}^{\prime \prime} \subset k_{0}^{\prime}$ then one can replace $k_{0}^{\prime}$ by $k_{0}^{\prime \prime}$ in the above formulation.

2. In the case when $\rho$ is trivial and the prehomogeneous space is obtained by reduction from characteristic zero, according to [4] one has $m=0$ in the identity (1.7). Also in this case $\zeta$ is a root of unity. We don't know whether this remains true for arbitrary representation $\rho$.

Corollary 1.3. Let $\chi$ be one of the characters $\lambda_{i}^{-1}$ or $\mu_{j}$. Then

$$
\left.\mathcal{F}\left(j_{! *}\left(L_{\chi}(f) \otimes \mathcal{V}_{\rho}[n]\right)\right)\right|_{U^{\vee}}=0 .
$$


Proof. This follows easily from the fact that the weight of the RHS in (1.7) drops for such $\chi$. Indeed, in this case the pure perverse sheaf of weight $2 n$

$$
\left.\mathcal{F}\left(j_{! *}\left(L_{\chi}(f) \otimes \mathcal{V}_{\rho}[n]\right)\right)\right|_{U \vee}
$$

is a quotient of the perverse sheaf

$$
\left.\mathcal{F}\left(j_{!}\left(L_{\chi}(f) \otimes \mathcal{V}_{\rho}[n]\right)\right)\right|_{U^{\vee}}
$$

which is pure of weight $<2 n$, hence the former sheaf should be zero.

As before we fix a square root of $\left|k_{0}\right|$ in $\overline{\mathbb{Q}}_{l}^{*}$. This allows to define the square root of the Tate twist $F \mapsto F\left(\frac{1}{2}\right)$ which multiplies the action of Frob $_{k_{0}}$ by $\left|k_{0}\right|^{-\frac{1}{2}}$.

Theorem 1.4. Keeping the notations of theorem 1.9 for every character $\chi$ of $\left(k_{0}^{\prime}\right)^{*}$ one has the following isomorphism of sheaves over $k_{0}^{\prime}$ :

$$
\begin{aligned}
& \widetilde{F}_{j_{! *}}\left(L_{\psi}\left(\frac{f(x) u_{1} \ldots u_{m}}{t_{1} \ldots t_{m+d}}\right) \otimes L_{\chi}\left(\frac{f(x) u_{1} \ldots u_{m}}{t_{1} \ldots t_{m+d}}\right) \otimes \mathcal{V}_{\rho}(x) \otimes\right. \\
& \left.\bigotimes_{i} L_{\lambda_{i}}\left(t_{i}^{-1}\right) \otimes \bigotimes_{j} L_{\mu_{j}}\left(u_{j}^{-1}\right)\right)\left(m+\frac{d+n}{2}\right) \simeq \zeta^{\operatorname{deg}} \otimes \widetilde{j}_{! *}^{\vee}\left(L_{\psi}\left(\frac{(-1)^{m+d} t_{1}^{\vee} \ldots t_{m+d}^{\vee}}{f^{\vee}\left(x^{\vee}\right) u_{1}^{\vee} \ldots u_{m}^{\vee}}\right) \otimes\right. \\
& \left.L_{\chi}\left(\frac{(-1)^{m+d} t_{1}^{\vee} \ldots t_{m+d}^{\vee}}{f^{\vee}\left(x^{\vee}\right) u_{1}^{\vee} \ldots u_{m}^{\vee}}\right) \otimes \mathcal{V}_{\rho \epsilon}\left(x^{\vee}\right) \otimes \bigotimes_{i} L_{\lambda_{i}}\left(-t_{i}^{\vee}\right) \otimes \bigotimes_{j} L_{\mu_{j}}\left(-u_{j}^{\vee}\right)\right)
\end{aligned}
$$

where $\tilde{j}: U \times \mathbb{G}_{m}^{2 m+d} \rightarrow V \times \mathbb{A}^{2 m+d}\left(\right.$ resp. $\tilde{j}^{\vee}: U^{\vee} \times \mathbb{G}_{m}^{2 m+d} \rightarrow V^{\vee} \times \mathbb{A}^{2 m+d}$ ) is the natural open embedding, $\left(x, t_{1}, \ldots, t_{m+d}, u_{1}, \ldots, u_{m}\right)$ are the coordinates on $V \times \mathbb{A}^{2 m+d},\left(x^{\vee}, t_{1}^{\vee}, \ldots, t_{m+d}^{\vee}, u_{1}^{\vee}, \ldots, u_{m}^{\vee}\right)$ are the dual coordinates on $V^{\vee} \times \mathbb{A}^{2 m+d}$, $\zeta^{\operatorname{deg}}$ is the geometrically constant sheaf of rank 1 on which Frob $k_{1}$ acts as $\zeta^{\left[k_{1}: k_{0}\right]}$, $\widetilde{j}_{!_{*}}$ denotes the Goresky-MacPherson extension (conjugated by an appropriate shift).

Remark. In the situation of the above theorem one can construct also the following isomorphism of sheaves:

$$
\begin{aligned}
& \mathcal{F} \widetilde{j}_{!_{*}}\left(L_{\psi}\left(\frac{f(x) u_{1} \ldots u_{m} t_{m+d}}{t_{1} \ldots t_{m+d-1}}\right) \otimes L_{\chi}\left(\frac{f(x) u_{1} \ldots u_{m}}{t_{1} \ldots t_{m+d-1}}\right) \otimes\right. \\
& \left.\mathcal{V}_{\rho}(x) \otimes \bigotimes_{i=1}^{m+d-1} L_{\lambda_{i}}\left(t_{i}^{-1}\right) \otimes \bigotimes_{j=1}^{m} L_{\mu_{j}}\left(u_{j}^{-1}\right)\right)\left(m+\frac{d+n}{2}\right) \simeq \zeta^{\mathrm{deg}} \otimes \\
& \widetilde{j}_{! *}^{\vee}\left(L_{\psi}\left(\frac{(-1)^{m+d} f^{\vee}\left(x^{\vee}\right) u_{1}^{\vee} \ldots u_{m}^{\vee} t_{m+d}^{\vee}}{t_{1}^{\vee} \ldots t_{m+d-1}^{\vee}}\right) \otimes L_{\lambda_{m+d}}\left(\frac{(-1)^{m+d} f^{\vee}\left(x^{\vee}\right) u_{1}^{\vee} \ldots u_{m}^{\vee} t_{m+d}^{\vee}}{t_{1}^{\vee} \ldots t_{m+d-1}^{\vee}}\right) \otimes\right. \\
& \left.\mathcal{V}_{\rho \epsilon}\left(x^{\vee}\right) \otimes \bigotimes_{i=1}^{m+d-1} L_{\lambda_{i}}\left(-t_{i}^{\vee}\right) \otimes \bigotimes_{j=1}^{m} L_{\mu_{j}}\left(-u_{j}^{\vee}\right) \otimes L_{\chi}\left(-t_{m+d}^{\vee}\right)\right)
\end{aligned}
$$

The proof is completely analogous to that of theorem 1.4.

\section{Auxiliary Results}

Lemma 2.1. For almost all $\chi$ the perverse sheaf $S_{V}(\chi, \rho)$ is irreducible. 
Proof. Consider the closed embedding $i: U \rightarrow V \times \mathbb{G}_{m}$ such that $i(x)=(x, f(x))$. Let $K=i_{*} \mathcal{V}_{\rho}$. Then according to [5] Theorem 2.3.1 (based on [8] (6.5.2)) the canonical map

$$
p_{1 !}\left(K \otimes p_{2}^{*} L_{\chi}\right) \rightarrow p_{1 *}\left(K \otimes p_{2}^{*} L_{\chi}\right)
$$

is an isomorphism for almost all $\chi$. Hence, the canonical map

$$
j_{!}\left(L_{\chi}(f) \otimes \mathcal{V}_{\rho}\right)[n] \rightarrow j_{*}\left(L_{\chi}(f) \otimes \mathcal{V}_{\rho}\right)[n]
$$

is an isomorphism for almost all $\chi$. Therefore, for almost all $\chi$ the sheaf $S_{V}(\chi, \rho)$ coincides with the Goresky-MacPherson extension of an irreducible perverse sheaf $L_{\chi}(f) \otimes \mathcal{V}_{\rho}[n]$.

Remark. To find explicitly the finite set of multiplicative characters $\chi$ for which $S_{V}(\chi, \rho)$ is not irreducible is a difficult problem. When $\rho=1$ this set can be found from the Bernstein polynomial of $f$ (see [4]). In general one can at least assert that if $S_{V}(\chi, \rho)$ is not irreducible then there exists a point $x \in V \backslash U$ such that $\left(\left.\alpha\right|_{H_{x}}\right)^{*} L_{\chi}$ is trivial, where $H_{x}$ is the stabilizer of $x$. In the cases when $G$-orbits on $V$ are known this allows to give a bound on the set of exceptional characters.

Lemma 2.2. Let $\mathcal{V}$ be an irreducible $G$-equivariant local system on $U$. Then there exists a multiplicative character of finite order $\chi_{\mathcal{V}}$ and a canonical isomorphism of $G$-equivariant sheaves on $\mathbb{G}_{m} \times U$ (where $G$ acts on the second factor):

$$
\operatorname{dil}^{*} \mathcal{V} \simeq L_{\chi \mathcal{V}} \otimes \mathcal{V}
$$

where dil : $\mathbb{G}_{m} \times U \rightarrow U$ maps $(t, x)$ to tx.

Proof. The local system $\operatorname{dil}^{*} \mathcal{V}$ on $\mathbb{G}_{m} \times U$ is $G$-equivariant with respect to the action of $G$ on the second factor, hence we have

$$
\operatorname{dil}^{*} \mathcal{V} \simeq L \otimes \mathcal{V}^{\prime}
$$

for some local system $L$ on $\mathbb{G}_{m}$ and some $G$-equivariant local system $\mathcal{V}^{\prime}$ on $U$. Restricting this isomorphism to $\{1\} \times U$ we find that $\mathcal{V}^{\prime} \simeq \mathcal{V}$ and the rank of $L$ is equal to 1 . It remains to prove that $L$ has form $L_{\chi_{\mathcal{V}}}$. To this end let us fix a point $x_{0} \in U$ and let $H_{l}$ be the stabilizer of the line $l=l_{x_{0}}$ spanned by $x_{0}$. Then we have a surjective homomorphism $\pi: H_{l} \rightarrow \mathbb{G}_{m}$, hence there exists a homomorphism $\sigma: \mathbb{G}_{m} \rightarrow H_{l}$ such that $\pi \circ \sigma: \mathbb{G}_{m} \rightarrow \mathbb{G}_{m}$ is of the form $t \mapsto t^{N}$ for some $N>0$. Now from $G$-equivariance we derive that the pull-back of $L$ by $\pi$ is trivial. Hence, $[\pi \circ \sigma]^{*} L$ is trivial, so $L \simeq L_{\chi \mathcal{V}}$ for some $\chi_{\mathcal{V}}$ of finite order.

Lemma 2.3. Let $K$ be a sheaf on $\mathbb{G}_{m}$ such that $H^{i}\left(\mathbb{G}_{m}, L_{\chi} \otimes K\right)=0$ for every multiplicative character $\chi$ and every $i \neq 0$. Then $K$ is perverse.

Proof. Since the functor $R \Gamma_{c}\left(\mathbb{G}_{m}, ?\right)$ has cohomological amplitude $[0,1]$ with respect to the perverse $t$-structure our assumption on $K$ immediately implies that ${ }^{p} H^{i} K=$ 0 for $i \neq-1,0$, while for any $\chi$ one has

$$
H_{c}^{0}\left(\mathbb{G}_{m}, L_{\chi} \otimes{ }^{p} H^{-1} K\right)=H_{c}^{1}\left(\mathbb{G}_{m}, L_{\chi} \otimes{ }^{p} H^{0} K\right)=0
$$

and

$$
H_{c}^{0}\left(\mathbb{G}_{m}, L_{\chi} \otimes K\right)=H_{c}^{1}\left(\mathbb{G}_{m}, L_{\chi} \otimes{ }_{6}^{p} H^{-1} K\right) \oplus H_{c}^{0}\left(\mathbb{G}_{m}, L_{\chi} \otimes{ }^{p} H^{0} K\right)
$$


This implies that $\chi\left(\mathbb{G}_{m},{ }^{p} H^{-1} K\right)=0$, hence $R \Gamma_{c}\left(\mathbb{G}_{m}, L_{\chi} \otimes{ }^{p} H^{-1} K\right)=0$ for every $\chi$. Applying theorem of Laumon (see Proposition 3.4.5 of [5]) we obtain that ${ }^{p} H^{-1} K=0$, so $K$ is perverse.

We say that a sheaf $K$ on $\mathbb{G}_{m}$ is !-hypergeometric if it is obtained as a multiplicative shift of the !-convolution of a finite number of sheaves of the form $\left.L_{\chi} \otimes L_{\psi}\right|_{\mathbb{G}_{m}}[1]$ or $\operatorname{inv}^{*}\left(\left.L_{\chi} \otimes L_{\psi}\right|_{\mathbb{G}_{m}}\right)[1]$ where inv : $\mathbb{G}_{m} \rightarrow \mathbb{G}_{m}: t \mapsto t^{-1}$. Recall (see e.g. Proposition 8.5.2 of [7]) that any perverse sheaf on $\mathbb{G}_{m}$ has non-negative Euler-Poincaré characteristic and the only simple perverse sheaves with zero Euler-Poincaré characteristic are the sheaves of the form $L_{\chi}[1]$. By a theorem of Katz every simple perverse sheaf with Euler-Poincaré characteristic equal to 1 is !-hypergeometric. The following proposition is a slight strengthening of this result.

Proposition 2.4. Let $K$ be a sheaf on $\mathbb{G}_{m}$ such that $\chi\left(\mathbb{G}_{m}, K\right)=1$ and $H_{c}^{i}\left(\mathbb{G}_{m}, L_{\chi} \otimes\right.$ $K)=0$ for $i \neq 0$ for every multiplicative character $\chi$. Then $K$ is a !-hypergeometric sheaf.

Proof. It follows from lemma 2.3 that $K$ is perverse. We use induction in length of $K$. If $K$ is simple then the assertion follows from Theorem 8.5.3 of [7]. Assume that $K$ is not simple and let $K_{0}$ be a simple perverse subobject of $K$, so that we have an exact sequence

$$
0 \rightarrow K_{0} \rightarrow K \rightarrow K_{1} \rightarrow 0 .
$$

We necessarily have $\chi\left(\mathbb{G}_{m}, K_{1}\right) \leq 1$. Suppose $\chi\left(\mathbb{G}_{m}, K_{1}\right)=0$. Then $K_{1}$ is a successive extension of sheaves of the form $L_{\chi}[1]$. However, for any $\chi$ one has

$$
\operatorname{Hom}\left(K, L_{\chi}[1]\right) \simeq H^{0}\left(\mathbb{G}_{m}, D\left(K \otimes L_{\chi^{-1}}[1]\right)\right) \simeq H_{c}^{0}\left(\mathbb{G}_{m}, K \otimes L_{\chi^{-1}}[1]\right)^{*}=0
$$

(where $D$ denotes the Verdier duality) which is a contradiction. Therefore, $\chi\left(\mathbb{G}_{m}, K_{1}\right)=$ 1. Furthermore, for any $\chi$ the $\operatorname{space} \operatorname{Hom}\left(K_{1}, L_{\chi}[1]\right)$ is a $\operatorname{subspace}$ of $\operatorname{Hom}\left(K, L_{\chi}[1]\right)=$ 0 . Thus, $H_{c}^{1}\left(\mathbb{G}_{m}, K_{1} \otimes L_{\chi^{-1}}\right)=0$ and by induction assumption $K_{1}$ is a !-hypergeometric sheaf. On the other hand, $\chi\left(\mathbb{G}_{m}, K_{0}\right)=0$, hence $K_{0} \simeq L_{\chi_{0}}[1]$ for some character $\chi_{0}$. Notice that the exact sequence (2.1) doesn't split (otherwise, we would have $\left.H_{c}^{1}\left(\mathbb{G}_{m}, L_{\chi_{0}^{-1}} \otimes K\right) \neq 0\right)$. It remains to use the fact that every non-trivial extension of a !-hypergeometric sheaf $K_{1}$ by $L_{\chi_{0}}[1]$ is !-hypergeometric which follows from the fact that $\operatorname{dim} \operatorname{Ext}^{1}\left(K_{1}, L_{\chi_{0}}[1]\right)=1$ and Theorem 8.4.7 of [7].

Lemma 2.5. Assume that for some collections of characters $\lambda_{i}, \mu_{j}, \lambda_{k}^{\prime}, \mu_{l}^{\prime}$ defined over finite field $k_{0}$ one has the identity

$$
\prod g\left(\chi\left(\lambda_{i} \circ N\right)\right) g\left(\chi^{-1}\left(\mu_{j} \circ N\right)\right)=c^{\left[k_{1}: k_{0}\right]} \chi(a) \prod g\left(\chi\left(\lambda_{k}^{\prime} \circ N\right)\right) g\left(\chi^{-1}\left(\mu_{l}^{\prime} \circ N\right)\right)
$$

for every multiplicative character $\chi$ of every finite extension $k_{1}$ of $k_{0}$, where $c \in \overline{\mathbb{Q}}_{l}^{*}$, $a \in k_{0}^{*}$ are some constants, $N: k_{1}^{*} \rightarrow k_{0}^{*}$ is the norm. Then the collections $\left(\lambda_{i}\right)$ and $\left(\lambda_{k}^{\prime}\right)$ (resp. $\left(\mu_{j}\right)$ and $\left.\left(\mu_{l}^{\prime}\right)\right)$ coincide.

Proof. Both parts of the identity are multiplicative Fourier transform of trace functions of !-hypergeometric sheaves on $\mathbb{G}_{m}$. Hence, by Theorem 1.1 .2 of [12] semisimplifications of these sheaves are isomorphic. Looking at the monodromy at 0 and $\infty$ we conclude that the corresponding collections of characters should coincide (see [7, Theorems 8.4.11 and 8.4.12). 
Lemma 2.6. Let $\chi$ be a non-trivial multiplicative character. Then for every sheaf $K$ on $\mathbb{A}^{1}$ one has a canonical isomorphism

$$
H_{c}^{0}\left(\mathbb{G}_{m}, L_{\chi} \otimes j_{1}^{*} \mathcal{F}(K)\right) \simeq G(\chi) \otimes H_{c}^{0}\left(\mathbb{G}_{m}, L_{\chi^{-1}} \otimes j_{1}^{*} K\right) .
$$

Proof. One has

$$
H_{c}^{0}\left(\mathbb{G}_{m}, L_{\chi} \otimes j_{1}^{*} \mathcal{F}(K)\right) \simeq H_{c}^{0}\left(\mathbb{G}_{m} \times \mathbb{A}^{1}, L_{\chi}(a) \otimes L_{\psi}(a t) \otimes p_{2}^{*} K[1]\right)
$$

where $a$ is the coordinate on $\mathbb{G}_{m}, t$ is the coordinate on $\mathbb{A}^{1}$. Projecting to the second factor and using (1.2) we obtain the result.

\section{Computation}

To compute the Fourier transform of $j_{!}\left(L_{\chi}(f) \otimes V_{\rho}\right)$ we want to combine the information coming from $G$-equivariance and from the stationary phase approximation. Technically the latter is obtained from the Picard-Lefschetz formula. The idea is to use the condition that $F=\operatorname{grad} \log f$ induces a birational isomorphism from $V$ to $V^{\vee}$. Let us define a rational function on $V \times \mathbb{A}^{d}$ where $d=\operatorname{deg} f$ by

$$
R\left(x, t_{1}, \ldots, t_{d}\right)=\frac{f(x)}{t_{1} \ldots t_{d}} .
$$

We will denote by $y=\left(x, t_{1}, \ldots, t_{d}\right)$ the coordinate in $V \times \mathbb{A}^{d}$ and by $\xi=$ $\left(x^{\vee}, t_{1}^{\vee}, \ldots, t_{d}^{\vee}\right)$ the coordinate in the dual space $V^{\vee} \times \mathbb{A}^{d}$. We denote

$$
\langle y, \xi\rangle=\left\langle x, x^{\vee}\right\rangle+t_{1} t_{1}^{\vee}+\ldots+t_{d} t_{d}^{\vee} .
$$

Lemma 3.1. The map $y \mapsto \operatorname{grad} R(y)$ induces an isomorphism from $U \times \mathbb{G}_{m}^{d}$ to $U^{\vee} \times \mathbb{G}_{m}^{d}$ with the inverse $\xi \mapsto(-1)^{d} R^{\vee}(\xi)^{-2} \operatorname{grad} R^{\vee}(\xi)$ where $R^{\vee}$ is defined in a similar way as $R$ starting from $f^{\vee}$ instead of $f$.

Proof. This follows easily from (1.3).

The above lemma implies that for $\xi \in U^{\vee} \times \mathbb{G}_{m}^{d}$ the function

$$
R_{\xi}(y)=R(y)+\langle y, \xi\rangle
$$

has a unique critical point in $U \times \mathbb{G}_{m}^{d}$, namely $y_{\xi}=(\operatorname{grad} R)^{-1}(\xi)$. Moreover, $y_{\xi}$ is a non-degenerate quadratic singularity of the corresponding level set of $R_{\xi}$ (see 15, sec. 6; in particular, if characteristics of $k$ is equal to 2 this implies that $n+d$ is even).

Lemma 3.2. The unique critical value of $R_{\xi}$ is equal to $(-1)^{d} R^{\vee}(\xi)^{-1}$.

Proof. According to lemma 3.1 the unique critical point of $R_{\xi}$ is

$$
y_{\xi}=(-1)^{d} R^{\vee}(\xi)^{-2} \operatorname{grad} R^{\vee}(\xi),
$$

hence

$$
\left\langle y_{\xi}, \xi\right\rangle=(-1)^{d} R^{\vee}(\xi)^{-2} \cdot\left\langle\operatorname{grad} R^{\vee}(\xi), \xi\right\rangle=0
$$

since $\operatorname{deg} R^{\vee}=0$. Hence, the critical value is equal to

$$
R_{\xi}\left(y_{\xi}\right)=R\left(y_{\xi}\right)=R\left(\operatorname{grad} R^{\vee}(\xi)\right) .
$$

Now using (1.4) it is easy to see that

$$
R\left(\operatorname{grad} R^{\vee}(\xi)\right)=(-1)^{d} R^{\vee}(\xi)^{-1} .
$$


Lemma 3.3. For every multiplicative character $\chi$, an irreducible $G$-equivariant local system $\mathcal{V}$ on $U$ and a point $\xi \in U^{\vee} \times \mathbb{G}_{m}^{d}$ one has

$$
\left.G\left(\chi^{-1}\right) \otimes \mathcal{F}\left(J_{!}\left(L_{\chi}(R) \otimes p_{U}^{*} \mathcal{V}\right)\right)\right|_{\xi} \simeq R \Gamma_{c}\left(\mathbb{G}_{m}, L_{\chi^{-1} \chi \mathcal{V}} \otimes j_{1}^{*} \mathcal{F}\left(R_{\xi !}\left(p_{U}^{*} \mathcal{V}\right)\right)[n+d]\right)
$$

where $J: U \times \mathbb{G}_{m}^{d} \rightarrow V \times \mathbb{A}^{d}$ is the natural embedding, $R_{\xi}: U \times \mathbb{G}_{m}^{d} \rightarrow \mathbb{A}^{1}$ is the morphism defined by (3.1), $p_{U}$ is the projection to $U$, the character $\chi \mathcal{V}$ was defined in lemma 2.2, $j_{1}: \mathbb{G}_{m} \rightarrow \mathbb{A}^{1}$ is the standard embedding.

Proof. Using the isomorphism (1.1) we can write

$$
\begin{aligned}
& \left.G\left(\chi^{-1}\right) \otimes \mathcal{F}\left(J_{!}\left(L_{\chi}(R) \otimes p_{U}^{*} \mathcal{V}\right)\right)\right|_{\xi} \simeq \\
& \simeq R \Gamma_{c}\left(\mathbb{G}_{m} \times U \times \mathbb{G}_{m}^{d}, L_{\chi^{-1}}(a) \otimes L_{\psi}(a R(y)+\langle y, \xi\rangle) \otimes p_{U}^{*} \mathcal{V}[n+d+1]\right)
\end{aligned}
$$

where $a$ is coordinate on the first factor $\mathbb{G}_{m}$. Now making a change of variables $y \mapsto a y$ and using the fact that $R$ has degree 0 and lemma 2.2 we obtain

$$
\begin{aligned}
& R \Gamma_{c}\left(\mathbb{G}_{m} \times U \times \mathbb{G}_{m}^{d}, L_{\chi^{-1}}(a) \otimes L_{\psi}(a R(y)+\langle y, \xi\rangle) \otimes p_{U}^{*} \mathcal{V}[n+d+1]\right) \\
& \left.\simeq R \Gamma_{c}\left(\mathbb{G}_{m} \times U \times \mathbb{G}_{m}^{d}, L_{\chi^{-1} \chi_{\mathcal{V}}}(a) \otimes L_{\psi}\left(a R_{\xi}(y)\right) \otimes p_{U}^{*} \mathcal{V}\right)[n+d+1]\right) \\
& \simeq R \Gamma_{c}\left(\mathbb{G}_{m} \times \mathbb{A}^{1}, p_{1}^{*} L_{\chi^{-1} \chi_{\mathcal{V}}} \otimes L_{\psi}(a t) \otimes p_{2}^{*} R_{\xi !}\left(p_{U}^{*} \mathcal{V}\right)[n+d+1]\right) \\
& \simeq R \Gamma_{c}\left(\mathbb{G}_{m}, L_{\chi^{-1} \chi_{\mathcal{V}}} \otimes j_{1}^{*} \mathcal{F}\left(R_{\xi !}\left(p_{U}^{*} \mathcal{V}\right)\right)[n+d]\right) .
\end{aligned}
$$

Lemma 3.4. For every $\xi \in U^{\vee} \times \mathbb{G}_{m}^{d}$ the sheaf $j_{1}^{*} \mathcal{F}\left(R_{\xi !}\left(p_{U}^{*} \mathcal{V}\right)\right)[n+d]$ on $\mathbb{G}_{m}$ is perverse.

Proof. The sheaf $\left(J^{\vee}\right)^{*} \mathcal{F}\left(J_{!}\left(L_{\chi}(R) \otimes p_{U}^{*} \mathcal{V}\right)\right)[n+d]$ on $U^{\vee} \times \mathbb{G}_{m}^{d}$ is perverse and relative equivariant with respect to the transitive action of $G \times \mathbb{G}_{m}$. Therefore, it is equal to some local system shifted by $[n+d]$. Now lemma 3.3 implies that for any $\chi$ the cohomology of $R \Gamma_{c}\left(\mathbb{G}_{m}, L_{\chi} \otimes j_{1}^{*} \mathcal{F}\left(R_{\xi !}\left(p_{U}^{*} \mathcal{V}\right)\right)[n+d]\right)$ is concentrated in degree zero. It remains to apply lemma 2.3.

Lemma 3.5. For every $\xi \in U^{\vee} \times \mathbb{G}_{m}^{d}$ and every $i \neq n+d$ the sheaf ${ }^{p} H^{i} R_{\xi !}\left(p_{U}^{*} \mathcal{V}\right)$ is constant (up to a shift).

Proof. This follows from lemma 3.4 and from $t$-exactness of the Fourier transform (see [12]).

Let $\mathcal{V}=\mathcal{V}_{\rho}$ be an irreducible $G$-equivariant local system on $U, \chi$ be a multiplicative character. The sheaf $F=j_{!}\left(L_{\chi}(f) \otimes \mathcal{V}\right)$ on $V$ is relative $G$-equivariant with characters $(\alpha, \chi)$. Therefore, its Fourier transform is also relative $G$-equivariant with the same characters. Taking into account the fact that $\mathcal{F}(F)[n]$ is perverse we conclude that

$$
\left(j^{\vee}\right)^{*} \mathcal{F}\left(j_{!}\left(L_{\chi}(f) \otimes \mathcal{V}\right)\right) \simeq L_{\chi^{-1}}\left(f^{\vee}\right) \otimes \mathcal{V}^{\vee}
$$

where $\mathcal{V}^{\vee}$ is a $G$-equivariant sheaf on $U^{\vee}$. Therefore, we have

$$
\left(J^{\vee}\right)^{*} \mathcal{F}\left(J_{!}\left(L_{\chi}(R) \otimes p_{U}^{*} \mathcal{V}\right)\right) \simeq G\left(\chi^{-1}\right)^{d} \otimes L_{\chi^{-1}}\left(R^{\vee}\right) \otimes p_{U^{\vee}}^{*} \mathcal{V}^{\vee}
$$

where $p_{U^{\vee}}: U^{\vee} \times \mathbb{G}_{m}^{d} \rightarrow U^{\vee}$ is the projection. Let us fix a point $\xi \in U^{\vee} \times \mathbb{G}_{m}^{d}$ such that $R^{\vee}(\xi)=1$. Then we have an isomorphism of $G\left(\chi^{-1}\right)^{d} \otimes \mathcal{V}_{\xi}^{\vee}$, where $\mathcal{V}_{\xi}^{\vee}$ is the 
fiber of $p_{U^{\vee}}^{*} \mathcal{V}^{\vee}$ at $\xi$, with the fiber of $\mathcal{F}\left(J_{!}\left(L_{\chi}(R) \otimes p_{U}^{*} \mathcal{V}\right)\right)$ at $\xi$. Using lemma 3.3 we can write

$$
\mathcal{V}_{\xi}^{\vee} \simeq G\left(\chi^{-1}\right)^{-d-1} \otimes R \Gamma_{c}\left(\mathbb{G}_{m}, L_{\chi^{-1} \chi \mathcal{V}} \otimes j_{1}^{*} \mathcal{F}\left(R_{\xi !}\left(p_{U}^{*} \mathcal{V}\right)\right)[n+d]\right)
$$

Notice that this isomorphism is compatible with the grading (where $\operatorname{deg} G\left(\chi^{-1}\right)=$ 0 ) so the cohomology of the RHS is concentrated in degree 0 .

Proof of theorem 1.1. Assume that $\chi \neq \chi \mathcal{V}$. Then using lemma 2.6 and lemma 3.5 we deduce from (3.2) a (non-canonical) isomorphism

$$
\mathcal{V}_{\xi}^{\vee} \simeq H_{c}^{0}\left(\mathbb{G}_{m}, L_{\chi \chi_{\mathcal{\nu}}^{-1}} \otimes K\right) \text {. }
$$

where $K=j_{1}^{* p} H^{n+d} R_{\xi !}\left(p_{U}^{*} \mathcal{V}\right)$. Let $H_{\xi} \subset G \times \mathbb{G}_{m}^{d}$ be the stabilizer of $\xi, \bar{H}_{\xi}$ be its group of connected components. The isomorphism (3.3) is compatible with the action of $\bar{H}_{\xi}$ (the action on the RHS is induced by the action of $H_{\xi}$ on $V \times \mathbb{G}_{m}^{d}$ which preserves the function $R_{\xi}$ ). We can write $K=\oplus_{W} W \otimes K_{W}$ where the sum is taken over irreducible representations of $\bar{H}_{\xi}, K_{W}$ are perverse sheaves on $\mathbb{G}_{m}$. Then we have

$$
\mathcal{V}_{\xi}^{\vee} \simeq \oplus_{W} W^{\oplus \chi_{c}\left(\mathbb{G}_{m}, K_{W}\right)}
$$

According to Grothendieck-Ogg-Shafarevich formula we have

$$
\chi_{c}\left(\mathbb{G}_{m}, K_{W}\right)=-\sum_{t \in \mathbb{G}_{m}(k)} a_{t}\left(K_{W}\right)-s_{0}\left(K_{W}\right)-s_{\infty}\left(K_{W}\right)
$$

where for every $t \in \mathbb{P}^{1}(k)$ we denote by $s_{t}(F)$ the Swan conductor of $F$ at $t$, $a_{t}(F)=\operatorname{rk}(F)+s_{t}(F)-\operatorname{rk}\left(\left.F\right|_{t}\right)$. Note that all the numbers $-a_{t}\left(K_{W}\right)$ are nonnegative since $K_{W}$ are perverse (see lemma (2.2.1.1) of [12]). Furthermore, we have

$$
-a_{t}\left(K_{W}\right) \geq \operatorname{rk}\left(K_{W}[-1]\right)-\operatorname{rk}\left(\left.K_{W}\right|_{t}[-1]\right)=\operatorname{rk} \Phi_{t}\left(K_{W}[-1]\right)
$$

where $\Phi_{t}$ denotes the vanishing cycles functor near the point $t$ (indeed, the restriction of $K_{W}[-1]$ to some non-empty open subset of $\mathbb{G}_{m}$ is a local system concentrated in degree 0 , hence $\left.s_{t}\left(K_{W}[-1]\right) \geq 0\right)$. Thus, we have an isomorphism of $\bar{H}_{\xi}$-representations

$$
\mathcal{V}_{\xi}^{\vee} \simeq \oplus_{t \in \mathbb{G}_{m}} \Phi_{t}(K[-1]) \oplus P
$$

for some $\bar{H}_{\xi}$-representation $P$. Note that by lemma 3.5 we have

$$
\Phi_{t}(K[-1])=\Phi_{t}\left(R_{\xi !}\left(p_{U}^{*} \mathcal{V}\right)[n+d-1]\right)
$$

According to lemmas 3.1 and 3.2 the function $R_{\xi}$ has a unique critical point $y_{0}$ and the corresponding critical value is $t_{0}=(-1)^{d}$. Since $y_{0}$ is a unique critical point of $R_{\xi}$ on $U \times \mathbb{G}_{m}^{d}$, it is preserved by the action of $H_{\xi}$. Hence the $H_{\xi}$-module $\Phi_{t_{0}}\left(R_{\xi !}\left(p_{U}^{*} \mathcal{V}\right)[n+d-1]\right)$ contains

$$
\left.\left.\Phi\left(p_{U}^{*} \mathcal{V}\left[n^{\prime}-1\right]\right)\right|_{y_{0}} \simeq \mathcal{V}_{y_{0}} \otimes \Phi\left(\overline{\mathbb{Q}}_{l}[n+d-1]\right)\right|_{y_{0}}
$$

as a direct summand, where $\Phi$ denotes the functor of vanishing cycles with respect to $R_{\xi}, \mathcal{V}_{y_{0}}$ is the fiber of $p_{U}^{*} \mathcal{V}$ at $y_{0}$. Furthermore, since $y_{0}$ is a non-degenerate singular point we have according to Picard-Lefschetz formula (see [3]) that $L=$ $\left.\Phi\left(\overline{\mathbb{Q}}_{l}[n+d-1]\right)\right|_{y_{0}}$ is a one-dimensional vector space concentrated in degree 0 . Note that the isomorphism of $H_{\xi}$ with $H_{y_{0}}$ induced by the action of $H_{\xi}$ on $U \times \mathbb{G}_{m}^{d}$ coincides with the isomorphism between $H_{p_{U} \vee(\xi)}$ and $H_{p_{U}\left(y_{0}\right)}$ considered before. Let us denote by $H$ any of these group. The $H$-representation $\mathcal{V}_{\xi}^{\vee}$ contains $\mathcal{V}_{y_{0}} \otimes L$ 
as a direct summand. The action of $H$ on $\mathcal{V}_{y_{0}}$ is given by the representation $\rho$ where $\mathcal{V}=\mathcal{V}_{\rho}$. On the other hand, the space of vanishing cycles $L$ has a canonical generator up to a sign. This implies that the action of $H$ on it is given by some character $\epsilon: H \rightarrow\{ \pm 1\}$. According to lemma 2 for almost all $\chi$ the local system $\mathcal{V}^{\vee}$ is irreducible, hence $\mathcal{V}_{\xi}^{\vee}$ coincides with its direct summand $\mathcal{V}_{y_{0}} \otimes L$, so we have $\mathcal{V}^{\vee} \simeq \mathcal{V}_{\rho \otimes \epsilon}$. This proves part (a) of Theorem (1.1).

Now invoking the isomorphism (3.2) which holds for all characters $\chi$ we deduce that

$$
j_{1}^{*} \mathcal{F}\left(R_{\xi !}\left(p_{U}^{*} \mathcal{V}\right)\right)[n+d]=(\rho \otimes \epsilon) \otimes M
$$

for some sheaf $M$ on $\mathbb{G}_{m}$ such that $R \Gamma_{c}\left(\mathbb{G}_{m}, L_{\chi} \otimes M\right)$ is concentrated in degree zero for all $\chi$, and $\chi\left(\mathbb{G}_{m}, M\right)=1$. By lemma $3.4 M$ is perverse, hence, applying proposition 2.4 we conclude that $M$ is a !-hypergeometric sheaf. Thus, for every character $\chi$ we have a canonical isomorphism

$\left(j^{\vee}\right)^{*} \mathcal{F} j_{!}\left(L_{\chi}(f) \otimes \mathcal{V}_{\rho}\right) \simeq G\left(\chi^{-1}\right)^{-d-1} \otimes H_{c}^{0}\left(\mathbb{G}_{m}, L_{\chi^{-1} \chi \mathcal{V}} \otimes M\right) \otimes L_{\chi^{-1}}\left(f^{\vee}\right) \otimes \mathcal{V}_{\rho \otimes \epsilon}$.

This finishes the proof of theorem (1.1).

An additional information that we get from the above argument is recorded in the following lemma.

Lemma 3.6. The sheaf $R_{\xi !}\left(p_{U}^{*} \mathcal{V}\right)$ is tamely ramified on $\mathbb{P}^{1}$ and is smooth on $\mathbb{G}_{m} \backslash$ $\left\{(-1)^{d}\right\}$.

Proof of theorem 1.9. Let $M$ be the !-hypergeometric sheaf defined by (3.4). If $\xi$ is a $k_{0}$-point then $M$ is defined over $k_{0}$. It follows that for some $a \in k^{*}$ the sheaf $[a]^{*} M$ (where $[a](b)=a b$ ) is isomorphic (forgetting the action of Frobenius) to the !-convolution of sheaves of the form $L_{\lambda_{i}} \otimes L_{\psi}[1], i=1, \ldots, m_{1}$, inv ${ }^{*}\left(L_{\mu_{j}} \otimes L_{\psi}\right)[1]$, $j=1, \ldots, m_{2}$, where $\lambda_{i}$ and $\mu_{j}$ are multiplicative characters of some finite extension $k_{0}^{\prime}$ of $k_{0}$. First we claim that $m_{1}=m_{2}+1$ and $a=(-1)^{m_{2}+d}$. Indeed, lemma 3.6 implies that $\mathcal{F}\left(j_{1} ! M\right)$ is tamely ramified on $\mathbb{P}^{1}$ and is smooth outside $(-1)^{d+1}$. But $\left.\mathcal{F}\left(j_{1} ! M\right)\right|_{\mathbb{G}_{m}}$ is the !-hypergeometric sheaf obtained by a multiplicative shift by $a^{-1}$ of the !-convolution of the sheaves $L_{\psi}[1], L_{\mu_{j}} \otimes L_{\psi}[1], j=1, \ldots m_{2}$, and $\operatorname{inv}^{*}\left(L_{\lambda_{i}} \otimes L_{\psi}\right)[1], i=1, \ldots, m_{1}$. According to Theorem 8.4.11 of [7] such a sheaf is tamely ramified only if $m_{1}=m_{2}+1$, and smooth outside $(-1)^{d+1}$ only if $(-1)^{m_{1}} a=$ $(-1)^{d+1}$, which proves our claim. Taking traces of Frobenius acting on both sides of (3.5) we get the equality of the form

$$
\begin{array}{r}
\mathcal{F}_{k_{1}} j_{!}\left(\operatorname{Tr}\left(\rho_{x}\right) \chi(f(x))\right)\left(x^{\vee}\right)=c^{\left[k_{1}: k_{0}\right]} \cdot \chi(-1)^{m_{2}+d} \times \\
\frac{\prod_{i=1}^{m_{2}+1} g\left(\chi^{-1}\left(\lambda_{i}^{\prime} \circ N\right)\right) \prod_{j=1}^{m_{2}} g\left(\chi\left(\mu_{j}^{\prime} \circ N\right)\right)}{g\left(\chi^{-1}\right)^{d+1}} \cdot \operatorname{Tr}\left((\rho \otimes \epsilon)_{x^{\vee}}\right) \chi^{-1}\left(f^{\vee}\left(x^{\vee}\right)\right)
\end{array}
$$

for every $x^{\vee} \in U^{\vee}\left(k_{1}\right)$ where $k_{1}$ is a finite extension of $k_{0}^{\prime}, N: k_{1}^{*} \rightarrow\left(k_{0}^{\prime}\right)^{*}$ is the norm, $\mathcal{F}_{k_{1}}$ is the discrete Fourier transform on $V\left(k_{1}\right)$ defined as follows:

$$
\mathcal{F}_{k_{1}}(\phi)\left(x^{\vee}\right)=(-1)^{n} q^{-\frac{n}{2}} \sum_{x \in V\left(k_{1}\right)} \phi(x) \psi\left(T\left\langle x, x^{\vee}\right\rangle\right)
$$

where $q=\left|k_{1}\right|, T: k_{1} \rightarrow k_{0}$ is the trace. Note that for generic $\chi$ the sheaf $j_{!} L_{\chi}(f) \otimes \mathcal{V}_{\rho}$ is pure of weight 0 . Since the Fourier-Deligne transform sends pure 
sheaves to pure sheaves raising weights by $n$ we find from (3.6) that the constant $c$ should be of weight $d-2 m_{2}$ relative to $\left|k_{0}\right|$.

It remains to get rid of the denominator in the RHS of (3.6), i.e. to prove that $d+1$ of the characters $\lambda_{i}^{\prime}$ are trivial. The idea is to compute the Fourier transform by a different method to obtain an equality similar to (3.6) but with the denominator in the RHS only containing Gauss sums of the form $g\left(\chi^{-1} \chi_{i}\right)$ with $\chi_{i}$ non-trivial. Then lemma 2.5 will imply that the denominator in (3.6) can be cancelled.

Let us fix non-trivial multiplicative characters $\chi_{1}, \ldots, \chi_{d}$ of $k_{0}^{\prime}$ and consider the smooth sheaf

$$
\widetilde{\mathcal{V}}=\mathcal{V} \otimes L_{\chi_{1}} \otimes \ldots L_{\chi_{d}}
$$

on $U \times \mathbb{G}_{m}^{d}$. We want to compute the Fourier transform of the sheaf $J_{!}\left(L_{\chi}(R) \otimes \widetilde{\mathcal{V}}\right)$ on $V_{d}=V \times \mathbb{A}^{d}$ using the Radon transform (see e.g. Lemma 3.3.9 of 四). Since $R$ has degree zero we have

$$
\mathcal{F} J_{!}\left(L_{\chi}(R) \otimes \widetilde{\mathcal{V}}\right) \simeq \mathcal{R} J_{!}\left(L_{\chi}(R) \otimes \widetilde{\mathcal{V}}\right):=p_{2 !}\left(p_{1}^{*} J_{!}\left(L_{\chi}(R) \otimes \widetilde{\mathcal{V}}\right) \otimes \omega\right)[n+d]
$$

where $\omega$ is the sheaf on $V_{d} \times V_{d}^{\vee}$ defined as the cone of the natural adjunction morphism $e_{!} e^{!} \overline{\mathbb{Q}}_{l} \rightarrow \overline{\mathbb{Q}}_{l}$ for the closed embedding $e$ of the affine hyperplane $Q=$ $\{(y, \xi):\langle y, \xi\rangle=1\}$ into $V_{d} \times V_{d}^{\vee}$. Note that the stabilizer $H_{\xi}$ of $\xi \in V_{d}^{\vee}$ acts on $V_{d}$ preserving $Q \cap p_{2}^{-1}(\xi)$, hence the sheaf $\omega_{\xi}:=\left.\omega\right|_{p_{2}^{-1}(\xi)}$ is $H_{\xi^{-}}$-equivariant and the isomorphism

$$
\left.\mathcal{F}\left(J_{!}\left(L_{\chi}(R) \otimes \widetilde{\mathcal{V}}\right)\right)\right|_{\xi} \simeq R \Gamma_{c}\left(U \times \mathbb{G}_{m}^{d}, L_{\chi}(R) \otimes \widetilde{\mathcal{V}} \otimes J^{*} \omega_{\xi}[n+d]\right)
$$

is compatible with the $H_{\xi}$-action. We can write

$$
R \Gamma_{c}\left(U \times \mathbb{G}_{m}^{d}, L_{\chi}(R) \otimes \widetilde{\mathcal{V}} \otimes J^{*} \omega_{\xi}[n+d]\right) \simeq R \Gamma_{c}\left(\mathbb{G}_{m}, L_{\chi}(R) \otimes \mathcal{K}_{\xi}\right)
$$

where $\mathcal{K}_{\xi}=R_{!}\left(\widetilde{\mathcal{V}} \otimes J^{*} \omega_{\xi}[n+d]\right)$. Choosing $\xi$ in the form $\left(x^{\vee} ; 1, \ldots, 1\right)$ where $f^{\vee}\left(x^{\vee}\right)=1$ we obtain that

$$
\left.\mathcal{F}\left(j_{!}\left(L_{\chi}(f) \otimes \mathcal{V}\right)\right)\right|_{x^{\vee}} \simeq \bigotimes_{i=1}^{d} G\left(\chi^{-1} \chi_{i}\right)^{-1} \otimes R \Gamma_{c}\left(\mathbb{G}_{m}, \mathcal{K}_{\xi}\right) .
$$

We have an $H_{\xi}$-isomorphism $\mathcal{K}_{\xi} \simeq(\rho \otimes \epsilon) \otimes K_{\xi}$ and by proposition 2.4 the sheaf $K_{\xi}$ is !-hypergeometric. Thus, we obtain the equality similar to (3.6) but with constant factor of the form

$$
(\widetilde{c})^{\left[k_{1}: k_{0}\right]} \cdot \chi(\widetilde{a}) \cdot \frac{\prod_{i=1}^{\tilde{m}_{1}} g\left(\chi^{-1}\left(\widetilde{\lambda}_{i} \circ N\right)\right) \prod_{j=1}^{\tilde{m}_{2}} g\left(\chi\left(\widetilde{\mu}_{j} \circ N\right)\right)}{\prod_{i=1}^{d} g\left(\chi^{-1}\left(\chi_{i} \circ N\right)\right)}
$$

Since the characters $\chi_{i}$ are non-trivial we can finish the proof of (1.7) by applying lemma 2.5.

It remains to prove the last statement of Theorem 1.2. Let us fix $\rho$. For every finite extension $k_{0}^{\prime} \subset k_{1}$ and a character $\chi$ of $k_{1}^{*}$ we can write the equality (1.7) in the form

$$
\left(j^{\vee}\right)^{*} \mathcal{F}_{k_{1}}\left(j ! \phi_{\chi}\right)=C_{\chi} \cdot \phi_{\chi}^{\vee}
$$

where

$$
\phi_{\chi}(x)=\operatorname{Tr}\left(\rho_{x}\right) \chi(f(x))
$$

for $x \in U\left(k_{1}\right)$,

$$
\phi_{\chi}^{\vee}\left(x^{\vee}\right)=\operatorname{Tr}\left((\rho \otimes \epsilon)_{x}\right) \chi^{-1}\left((-1)^{m} f^{\vee}\left(x^{\vee}\right)\right)
$$


for $x^{\vee} \in U^{\vee}\left(k_{1}\right)$,

$$
C_{\chi}=\zeta^{\left[k_{1}: k_{0}\right]} \cdot \prod_{i=1}^{m+d} \frac{g\left(\chi\left(\lambda_{i} \circ N\right)\right)}{\sqrt{q}} \cdot \prod_{j=1}^{m} \frac{g\left(\chi^{-1}\left(\mu_{j} \circ N\right)\right)}{\sqrt{q}} .
$$

We want to prove that if $\chi$ is different from all the characters $\lambda_{i} \circ N, \mu_{j} \circ N$ then $\mathcal{F}_{k_{1}}\left(j_{!} \phi_{\chi}\right)$ is zero outside $U^{\vee}\left(k_{1}\right)$. First we claim that one can assume $k_{1}$ to be sufficiently large. Indeed, assume that there exists a finite extension $k_{1} \subset k_{1}^{\prime}$ such that for every finite extension $k_{1}^{\prime} \subset k_{2}$ the function $\mathcal{F}_{k_{2}}\left(j ! \phi_{\chi \circ N_{k_{2} / k_{1}}}\right)$ vanishes outside $U^{\vee}\left(k_{2}\right)$. This implies that the sheaf $\mathcal{F}\left(j_{!} L_{\chi}\right)$ is zero outside $U^{\vee}$ (by Theorem 1.1.2 of [12]), in particular, $\mathcal{F}_{k_{1}}\left(j_{!} \phi_{\chi}\right)$ vanishes outside $U^{\vee}\left(k_{1}\right)$. Now we can choose $k_{1}$ large enough so that there exists a character $\chi_{0}$ of $k_{1}^{*}$ (different from all the characters $\lambda_{i} \circ N$ and $\mu_{j} \circ N$ ) such that

$$
\mathcal{F}_{k_{1}}\left(j_{!} \phi_{\chi_{0}}\right)=C_{\chi_{0}} \cdot j_{!}^{\vee} \phi_{\chi_{0}}^{\vee}
$$

(this follows from theorem 1.1 (a)). Using the identity $\left\|\mathcal{F}_{k_{1}}(\phi)\right\|=\|\phi\|$ we derive that

$$
\left\|j_{!} \phi_{\chi_{0}}\right\|=\left|C_{\chi_{0}}\right| \cdot|| j_{!}^{\vee} \phi_{\chi_{0}}^{\vee} \|
$$

Now the statement follows imeediately from the fact that the norms $\left\|j_{!} \phi_{\chi}\right\|$ and $\left\|j_{!}^{\vee} \phi_{\chi}^{\vee}\right\|$ do not depend on $\chi$. Indeed, assume that $\chi$ is different from all the characters $\lambda_{i} \circ N$ and $\mu_{j} \circ N$. Then we have $\left|C_{\chi}\right|=\left|C_{\chi_{0}}\right|$, so we can replace $\chi_{0}$ by $\chi$ in the equality (3.8). It follows that the norm of the function $\mathcal{F}_{k_{1}}\left(j_{!} \phi_{\chi}\right)$ is equal to the norm of its restriction to $U^{\vee}\left(k_{1}\right)$, hence this function vanishes outside $U^{\vee}\left(k_{1}\right)$.

Proof of theorem 1.4. By irreducibility it suffices to prove the isomorphism of restrictions of sheaves in both sides to $U^{\vee} \times \mathbb{G}_{m}^{2 m+d}$. The idea is to combine (1.7) with the simple identity

$$
(1-q) \cdot \psi(a) \lambda(a)=\sum_{\nu} g(\lambda \nu) \nu^{-1}(a)
$$

where $a \in k_{1}^{*}, \lambda$ is a character of $k_{1}^{*}$, the sum is taken over all characters of $k_{1}^{*}$, by abuse of notation we denote by $\psi$ the additive character of $k_{1}$ composed of the trace and $\psi$. Applying (3.9) we get

$$
\begin{aligned}
& (1-q) \cdot\left(\widetilde{j}^{\vee}\right)^{*} \mathcal{F}_{k_{1}} \widetilde{j}_{!}\left(\psi\left(\frac{f(x) u_{1} \ldots u_{m}}{t_{1} \ldots t_{m+d}}\right)(\chi \circ N)\left(\frac{f(x) u_{1} \ldots u_{m}}{t_{1} \ldots t_{m+d}}\right) \operatorname{Tr}\left(\rho\left(r_{x}\right)\right) \times\right. \\
& \left.\prod_{i} \lambda_{i}^{-1}\left(N t_{i}\right) \prod_{j} \mu_{j}^{-1}\left(N u_{j}\right)\right)=\sum_{\nu} g(\nu(\chi \circ N)) \cdot\left(\widetilde{j}^{\vee}\right)^{*} \mathcal{F}_{k_{1}} \widetilde{j}_{!}\left(\nu^{-1}(f(x)) \operatorname{Tr}\left(\rho\left(r_{x}\right)\right) \times\right. \\
& \left.\prod_{i}\left(\nu\left(\lambda_{i}^{-1} \circ N\right)\right)\left(t_{i}\right) \prod_{j}\left(\nu\left(\mu_{j} \circ N\right)\right)^{-1}\left(u_{j}\right)\right) .
\end{aligned}
$$

Here $\mathcal{F}_{k_{1}}$ denotes the Fourier transform on $\left(V \times \mathbb{A}^{2 m+d}\right)\left(k_{1}\right)$ defined by a formula similar to (3.7) but with $n$ replaced by $n+2 m+d, N: k_{1}^{*} \rightarrow\left(k_{0}^{\prime}\right)^{*}$ is the norm. 
Now applying (1.7) we can rewrite this as

$$
\begin{aligned}
& \zeta^{\left[k_{1}: k_{0}\right]} \cdot q^{-2 m-d} \cdot \sum_{\nu} g(\nu(\chi \circ N)) \nu\left((-1)^{m} \frac{f^{\vee}\left(x^{\vee}\right) u_{1}^{\vee} \ldots u_{m}^{\vee}}{t_{1}^{\vee} \ldots t_{m+d}^{\vee}}\right) \operatorname{Tr}\left((\rho \epsilon)\left(r_{x^{\vee}}\right)\right) \times \\
& \prod_{i} \lambda_{i}\left(N t_{i}^{\vee}\right) g\left(\nu^{-1}\left(\lambda_{i} \circ N\right)\right) g\left(\nu\left(\lambda_{i}^{-1} \circ N\right)\right) \prod_{j} \mu_{j}\left(N u_{j}^{\vee}\right) g\left(\nu\left(\mu_{j} \circ N\right)\right) g\left(\nu^{-1}\left(\mu_{j} \circ N\right)^{-1}\right)= \\
& \zeta^{\left[k_{1}: k_{0}\right]} \cdot \sum_{\nu} g(\nu(\chi \circ N)) \nu\left(\frac{(-1)^{m+d} f^{\vee}\left(x^{\vee}\right) u_{1}^{\vee} \ldots u_{m}^{\vee}}{t_{1}^{\vee} \ldots t_{m+d}^{\vee}}\right) \operatorname{Tr}\left((\rho \epsilon)\left(r_{x^{\vee}}\right)\right) \times \\
& \prod_{i} \lambda_{i}\left(N\left(-t_{i}^{\vee}\right)\right) \prod_{j} \mu_{j}\left(N\left(-u_{j}^{\vee}\right)\right)+(1-q) \cdot \sum_{i=1}^{M} m_{i} t_{k_{1}}\left(F_{i}\right)
\end{aligned}
$$

where the coefficients $m_{i} \in \mathbb{Z}$ do not depend on the extension $k_{1}, t_{k_{1}}\left(F_{i}\right)$ are the trace functions of some simple perverse sheaves which are relative equivariant with respect to the natural action of $\mathbb{G}_{m} \times \mathbb{G}_{m}^{2 m+d}$ on $U \times \mathbb{G}_{m}^{2 m+d}$ (where the first factor $\mathbb{G}_{m}$ acts on $U$ by dilations). Finally we can use (3.9) again to deduce the following equality

$$
\begin{aligned}
& \left(\widetilde{j}^{\vee}\right)^{*} \mathcal{F}_{k_{1}} \widetilde{j}_{!}\left(\psi\left(\frac{f(x) u_{1} \ldots u_{m}}{t_{1} \ldots t_{m+d}}\right)(\chi \circ N)\left(\frac{f(x) u_{1} \ldots u_{m}}{t_{1} \ldots t_{m+d}}\right) \operatorname{Tr}\left(\rho\left(r_{x}\right)\right) \times\right. \\
& \left.\prod_{i} \lambda_{i}^{-1}\left(N t_{i}\right) \prod_{j} \mu_{j}^{-1}\left(N u_{j}\right)\right)= \\
& =\zeta^{\left[k_{1}: k_{0}\right]} \psi\left(\frac{(-1)^{m+d} t_{1}^{\vee} \ldots t_{m+d}^{\vee}}{f^{\vee}\left(x^{\vee}\right) u_{1}^{\vee} \ldots u_{m}^{\vee}}\right)(\chi \circ N)\left(\frac{(-1)^{m+d} t_{1}^{\vee} \ldots t_{m+d}^{\vee}}{f^{\vee}\left(x^{\vee}\right) u_{1}^{\vee} \ldots u_{m}^{\vee}}\right) \operatorname{Tr}\left((\rho \epsilon)\left(r_{x} \vee\right)\right) \times \\
& \prod_{i} \lambda_{i}\left(N\left(-t_{i}^{\vee}\right)\right) \prod_{j} \mu_{j}\left(N\left(-u_{j}^{\vee}\right)\right)+\sum_{i=1}^{M} m_{i} t_{k_{1}}\left(F_{i}\right) .
\end{aligned}
$$

The LHS of this identity is the trace function of the sheaf

$$
\left(\widetilde{j}^{\vee}\right)^{*} \mathcal{F} \widetilde{j}_{!}\left(L_{\psi}\left(\frac{f(x) u_{1} \ldots u_{m}}{t_{1} \ldots t_{m+d}}\right) \otimes \mathcal{L}\right)
$$

where

$$
\mathcal{L}=L_{\chi}\left(\frac{f(x) u_{1} \ldots u_{m}}{t_{1} \ldots t_{m+d}}\right) \otimes \mathcal{V}_{\rho}(x) \otimes \bigotimes_{i} L_{\lambda_{i}^{-1}}\left(t_{i}\right) \otimes \bigotimes_{j} L_{\mu_{j}^{-1}}\left(u_{j}\right) .
$$

Hence, it differs from the trace function of the sheaf

$$
F=\left(\widetilde{j}^{\vee}\right)^{*} \widetilde{\mathcal{F}}_{!_{*}}\left(L_{\psi}\left(\frac{f(x) u_{1} \ldots u_{m}}{t_{1} \ldots t_{m+d}}\right) \otimes \mathcal{L}\right)
$$

by a linear combination of trace functions of simple perverse sheaves (up to shift) on $U^{\vee} \times \mathbb{G}^{2 m+d}$ which are not isomorphic to $F$. Assume that $F \neq 0$. Then it is a simple perverse sheaf (up to shift), hence by Theorem 1.1.2 of [12] it coincides with one of the sheaves corresponding to terms in the RHS. It remains to prove that $F \neq 0$ and $F$ is not relative equivariant with respect to the action of $\mathbb{G}_{m} \times \mathbb{G}_{m}^{2 m+d}$. Note that the sheaf $F$ is the image of the morphism of perverse sheaves $F_{!} \rightarrow F_{*}$ where $F_{!}$(resp. $F_{*}$ ) is defined by the same formula but using the !-extension (resp. *-extension). Let us consider the action of $\mathbb{G}_{m}$ on $V \times \mathbb{A}^{2 m+d}\left(\right.$ resp. on $V^{\vee} \times \mathbb{A}^{2 m+d}$ ) such that for $t \in \mathbb{G}_{m}$ the corresponding operator $\beta(t): V \times \mathbb{A}^{2 m+d} \rightarrow V \times \mathbb{A}^{2 m+d}$ $\left(\right.$ resp. $\beta^{\vee}(t): V^{\vee} \times \mathbb{A}^{2 m+d} \rightarrow V^{\vee} \times \mathbb{A}^{2 m+d}$ ) multiplies the coordinate $t_{1}$ (resp. $t_{1}^{\vee}$ ) 
by $t$ and leaves all the other coordinates unchanged. Let us denote by $Q$ the map from $U \times \mathbb{G}_{m}^{2 m+d}$ to $\mathbb{G}_{m}$ given by

$$
Q\left(x, t_{1}, \ldots, t_{m+d}, u_{1}, \ldots, u_{m}\right)=\frac{f(x) u_{1} \ldots u_{m}}{t_{1} \ldots t_{m+d}} .
$$

Let us fix $\xi \in U^{\vee} \times \mathbb{G}_{m}^{2 m+d}$ and denote by $Q_{\xi}: U \times \mathbb{G}_{m}^{2 m+d} \rightarrow \mathbb{A}^{1}$ the map given by

$$
Q_{\xi}(\widetilde{x})=Q(\widetilde{x})+\langle\widetilde{x}, \xi\rangle \text {. }
$$

Then we claim that there exist a commutative diagram

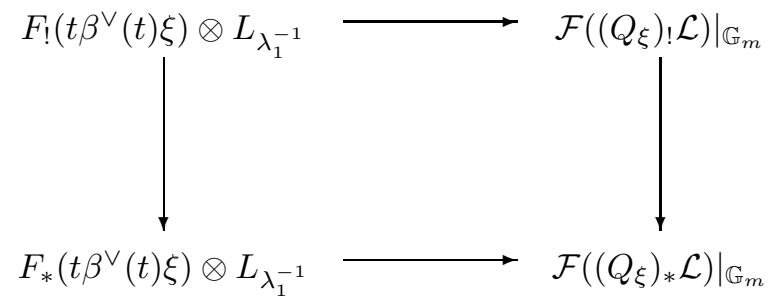

where rows are isomorphisms, $t$ is the coordinate on $\mathbb{G}_{m}$. Indeed, the first row is obtained from the following sequence of isomorphisms:

$$
\begin{aligned}
& \left.\mathcal{F}\left(\left(Q_{\xi}\right) ! \mathcal{L}\right)\right|_{t} \simeq H_{c}^{*}\left(U \times \mathbb{G}_{m}^{2 m+d}, L_{\psi}\left(t Q_{\xi}(\widetilde{x})\right) \otimes \mathcal{L}(\widetilde{x})\right) \simeq \\
& \simeq H_{c}^{*}\left(U \times \mathbb{G}_{m}^{2 m+d}, L_{\psi}\left(Q\left(\beta(t)^{-1} \widetilde{x}\right)+\langle\widetilde{x}, t \xi\rangle\right) \otimes \mathcal{L}(\widetilde{x})\right) \simeq \\
& \simeq H_{c}^{*}\left(U \times \mathbb{G}_{m}^{2 m+d}, L_{\psi}(Q(\widetilde{x})+\langle\beta(t) \widetilde{x}, t \xi\rangle) \otimes \mathcal{L}(\beta(t) \widetilde{x})\right) \simeq \\
& \left.\simeq H_{c}^{*}\left(U \times \mathbb{G}_{m}^{2 m+d}, L_{\psi}\left(Q(\widetilde{x})+\left\langle\widetilde{x}, t \beta^{\vee}(t) \xi\right\rangle\right) \otimes \mathcal{L}(\widetilde{x})\right) \otimes L_{\lambda_{1}^{-1}}\right|_{t} \simeq \\
& \left.\simeq F_{!}\left(t \beta^{\vee}(t) \xi\right) \otimes L_{\lambda_{1}^{-1}}\right|_{t} .
\end{aligned}
$$

Similarly, one constructs the lower arrow in the diagram (3.10) and the commutativity can be easily checked. Thus, it suffices to prove that the image of the morphism

$$
\left(Q_{\xi}\right) ! \mathcal{L} \rightarrow\left(Q_{\xi}\right)_{*} \mathcal{L}
$$

is not a smooth sheaf on $\mathbb{G}_{m}$. By exactness of the vanishing cycles functor it suffices to prove that for some point $t_{0} \in \mathbb{G}_{m}$ the natural map

$$
\Phi_{t_{0}}\left(Q_{\xi}\right) ! \mathcal{L} \rightarrow \Phi_{t_{0}}\left(Q_{\xi}\right)_{*} \mathcal{L}
$$

is non-zero, where $\Phi_{t_{0}}$ denotes the vanishing cycles functor at $t_{0}$. This follows from the fact that there exists a unique non-degenerate critical point of $Q_{\xi}$ in $U \times \mathbb{G}_{m}^{2 m+d}$ with non-zero critical value $t_{0}$ (this can be checked as in lemmas 3.1 and 3.2) and Picard-Lefschetz formula. Indeed, according to this formula the non-degenerate critical point gives a one-dimensional direct summand in both $\Phi_{t_{0}}\left(\left(Q_{\xi}\right) ! \mathcal{L}\right)$ and $\Phi_{t_{0}}\left(\left(Q_{\xi}\right)_{*} \mathcal{L}\right)$ which maps identically under the morphism 3.11$)$.

Acknowledgment. We are grateful to Pavel Etingof for helpful discussions.

\section{REFERENCES}

[1] A. Beilinson, J. Bernstein, P. Deligne, Faisceaux pervers, Asterisque 100 (1982), 7-172.

[2] P. Deligne, La conjecture de Weil, II, Publ. Math. IHES 52 (1980), 313-428.

[3] P. Deligne, La formule de Picard-Lefschetz, exp. XV in Groupes de monodromie en géométrie algébrique (SGA 7 II). Lecture Notes in Mathematics, Vol. 340. Springer-Verlag, Berlin-New York, 1973. 
[4] J. Denef, A. Gyoja, Character sums associated to prehomogeneous vector spaces, Compositio Math. 113 (1998), 273-346.

[5] O. Gabber, F. Loeser, Faisceaux pervers l-adique sur un tore, Duke Math. Journal 83 (1996), 501-606.

[6] L. Illusie, Théorie de Brauer et charactéristique d'Euler-Poincaré (d'après P. Deligne), in Caractéristique d'Euler-Poincaré, Asterisque 82-83 (1981), 161-172.

[7] N. Katz, Exponential sums and differential equations, Princeton University Press, 1990.

[8] N. Katz, G. Laumon, Transformation de Fourier et majoration de sommes exponentielles, Publ. IHES 62 (1986), 361-418.

[9] T. Kimura, T. Kogiso, M. Fujinaga, Fundamental theorem of prehomogeneous vector spaces of characteristic p, Bull. Austral. Math. Soc. 56 (1997), 331-341.

[10] T. Kimura, M. Sato, A classification of irreducible prehomogeneous vector spaces and their relative invariants, Nagoya Math. J. 65 (1977), 1-155.

[11] G. Laumon, Comparaison de caractéristiques d'Euler-Poincaré en cohomologie l-adique, C.R. Acad. Sci. Paris Ser. I Math. 292 (1981), 209-212.

[12] G. Laumon, Transformation de Fourier, constantes d'équations fonctionnelles et conjecture de Weil, Publ. IHES 65 (1987), 131-210.

[13] G. Lusztig, Intersection cohomology complexes on a reductive group, Invent. Math. 75 (1984), 205-272.

[14] G. Lusztig, Character sheaves. I, Adv. in Math. 56 (1985), 193-237.

[15] D. S. Rim, Formal deformation theory, exp. VI in Groupes de monodromie en géométrie algébrique (SGA 7 I). Lecture Notes in Mathematics, Vol. 288. Springer-Verlag, Berlin-New York, 1972

[16] M. Sato, Theory of prehomogeneous vector spaces (algebraic part)—the English translation of Sato's lecture from Shintani's note. Notes by T. Shintani. Translated from the Japanese by M. Muro. Nagoya Math. J. 120 (1990), 1-34. 